\title{
RENDIMIENTO POTENCIAL DEL CULTIVO DE PAPA EN BALCARCE, CAUSAS QUE LIMITAN LA PRODUCTIVIDAD REAL
}

\author{
Silvia T. Cantos de Ruiz*; Fernando H. Andrade**; Américo \\ Mendiburu**
}

\begin{abstract}
RESUMEN
Se calculó el rendimiento potencial del cultivo de papa en Balcarce (Buenos Aires, Argentina, latitud $37^{\circ} 45^{\prime} \mathrm{S}$ ), y se analizaron las causas de las diferencias entre el mencionado rendimiento y el real. El rendimiento potencial se estimó según el modelo de Van der Zaag. El rendimiento real se estudió en tres fechas de plantación (29/09, 10/10 y 10/11/86) en dos localidades del Partido de Balcarce. El rendimiento potencial promedio fue de 88 t.ha-1 de tubérculo en peso fresco. La fecha intermedia de plantación alcanzó los mayores rendimientos reales, estos llegaron, aproximadamente al 50\% del valor del rendimiento potencial. Las diferencias entre ambos rendimientos estuvieron dadas por deficiencias de intercepción de radiación fotosintéticamente activa y en productividad de follaje. Las fechas temprana y tardía tuvieron menores rendimientos en tubérculos debido principalmente a mayores limitaciones en productividad de follaje y en intercepción de radiación, respectivamente.
\end{abstract}

Palabras Claves Adicionales: Intercepción de la luz, índice de área foliar, producción de materia seca, fecha de plantación.

\section{ABSTRACT}

Potential Yield of the Potato Crop in Balcarce. Limitations on the Actual Productivity

The potential yield of the potato crop in Balcarce (Buenos Aires, Aceptado para publicación: 30/03/89.

* Ing. Agr. M.S. Facultad de Agronomía y Agroindustrias. U.N.S.E. Avda. Belgrano (S) 1912, (4200) Santiago del Estero, República Argentina.

** Ing. Agr. Ph.D. INTA. Estación Experimental Agropecuaria Balcarce. C.C. 276

(7620) Balcarce, Buenos Aires, República Argentina 
Argentina, Lat. $\left.37^{\circ} 45^{\prime} \mathrm{S}\right)$ was calculated, and the reasons determining the differences between the actual and potential yields were investigated. The potential yield was estimated following the Van der Zaag model. Actual yields were determined for three planting dates (September 29, October 10 and November 11, 1986) in two locations within the Balcarce County. The potential yield was $88 \mathrm{t}$ /ha of tuber fresh weight. The best actual yields were obtained in the second planting date. These yields were approximately one half of the potential yield. The differences between the actual and potential yields was due to defficient photosynthetically active radiation interception and foliage productivity. The first and third planting dates presented lower actual yields due to further limitations in foliage productivity and photosynthetically active radiation interception, respectively.

Additional Index Words: Light interception, leaf area index, dry matter production, planting date.

El Partido de Balcarce es el principal productor de papa del Sudeste de la provincia de Buenos Aires (SERBA). Esta zona aporta el $90 \%$ de la producción de papa semitardía en la República Argentina (6). Desde $1949 / 50$ la superficie plantada ha ido disminuyendo, sin embargo, la producción total y el rendimiento del cultivo han experimentado importantes incrementos anuales como resultado de la creciente incorporación de tecnologías adecuadas.

Los rendimientos reales promedio en la zona productora del SERBA se aproximan a $30 \mathrm{t}$ de tubérculos en peso fresco por hectárea; aunque algunos productores obtienen promedios de hasta $60 \mathrm{t} / \mathrm{ha}$.

Los datos de ensayos sugieren que los cultivos poseen deficiente cobertura durante el ciclo y que en varias situaciones los riegos aplicados no alcanzan a cubrir la demanda atmosférica (Suero, Comunicación Personal). Se supone entonces, que los rendimientos están limitados por ineficiencia en la interceptación y utilización de la radiación incidente. Teniendo en cuenta esta hipótesis, es conveniente llevar a cabo investigaciones tendientes a obtener información sobre el rendimiento potencial del cultivo de la papa en Balcarce, y a determinar las causas que limitan la producción real.

Zaag y Burtonn (21) y Zaag (19) desarrollaron un modelo para el cálculo del rendimiento potencial del cultivo de papa en función de radiación y temperatura, sobre la base de trabajos anteriores de de Wit (18); Sibma (15, 16); Goundriaan y Van Laar (9), entre otros. Esta estimación del rendimiento potencial, junto con ciertos datos de 
cultivos reales, proveen información sobre los factores que limitan los rendimientos y sobre el nivel técnico de producción de una determinada zona; ayudan además a generar pautas para optimizar el manejo y el mejoramiento genético del cultivo.

Los objetivos del presente trabajo fueron los siguientes:

1. Estimar el rendimiento potencial del cultivo de papa cv Huinkul MAG en Balcarce.

2. Calcular el rendimiento esperado sobre la base de valores reales de intercepción de radiación.

3. Medir el rendimiento real mediante cosechas sucesivas.

4. Determinar las causas de las diferencias entre el rendimiento real, el esperado para una condición de intercepción dada y el Potencial.

\section{MATERIALES Y MÉTODOS}

Para el cálculo del rendimiento potencial tanto promedio histórico (RPP) como anual (RPA) y rendimiento esperado para una condición de cobertura real (RE) se tomó como guía el modelo propuesto por Zaag y Burton (21) y validado por Zaag (19). El cálculo del RPP se basó sobre parámetros climáticos mensuales promedio de 17 años (período 1970-1987), mientras que el del RPA consideró los mismos parámetros para el ciclo 1986-1987 (Figura 1).

El cálculo del rendimiento potencial se realizó para un cultivo óptimo plantado el 19/10 (fecha óptima).

Para las radiaciones que llegan a Balcarce (37e 45' S) se estimó la producción diaria bruta del cultivo $(P)$ tanto para días claros $(P e)$ como para días nublados (Po), utilizando la tabla preparada por Goundriaan y Van Laar (9) (Amax $\left.=30 \mathrm{~kg} \mathrm{CO}_{2} \mathrm{ha}^{-1} \mathrm{~h}^{-1}\right)$. Para estimar la proporción del tiempo con cielo nublado se utilizó el porcentaje de Heliofonía relativa promedio mensual (He), en lugar del factor propuesto por Zaag.

$$
\mathrm{P}=(1-\% \mathrm{He}) \times \mathrm{Po}+\mathrm{He} \times \mathrm{Pc}
$$




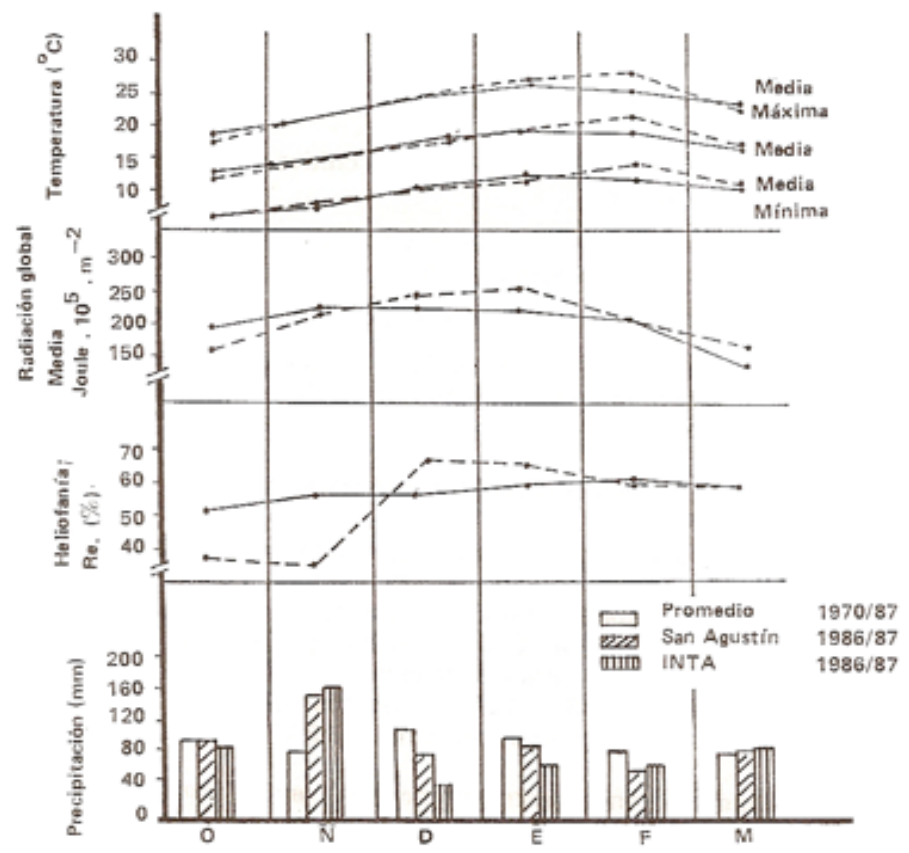

FIGURA 1. Evolución de parámetros climáticos en Balcarce durante el ciclo del cultivo.

Promedios mensuales del período 1970/1987 (-).

Promedios mensuales del ciclo 1986/1987 (---) 
El coeficiente de pérdidas por respiración ( $k$ ) necesario para estimar productividad neta ( $P$ neta) se calculó en función de las temperaturas medias máximas y mínimas mensuales (21).

$$
\mathrm{P} \text { neta }=\mathrm{P} \times \mathrm{k}
$$

La producción diaria para un cultivo de determinada cobertura (A) se obtuvo multiplicando $P$ neta por el porcentaje de radiación fotosintéticamente activa interceptada por el cultivo (\% Int) $(1,11)$.

$$
A=P \text { neta } \times \% \text { Int. }
$$

El \% Int. para el cultivo óptimo fue estimado (Butzonitch, Elverdín, Espinillo y Huarte, Comunicación Personal), multiplicando el valor $A$ para cada mes por los días del mes y sumándolos se obtuvo el valor del rendimiento potencial anual (RPA) o promedio (RPP).

Para el cálculo del rendimiento en tubérculo se tomó un índice de cosecha (IC) de $0.8(15,19,20)$ y se consideró un contenido de materia seca en los tubérculos de $20 \%$ (21).

El cálculo del RE se realizó en forma similar aunque se utilizaron valores reales de \% Int. Durante el ciclo del cultivo, se midió radiación fotosintéticamente activa (RFA) con una barra LI-COR 191 -SB de 1 $\mathrm{m}$ de longitud, conectada a un radiómetro LI-COR 188-B tanto arriba del cultivo (lo) como en el suelo (I). A las horas 10:30 y 14:00 en días despejados se tomaron de 50 a 80 lecturas por sitio experimental y se determinó el porcentaje de intercepción mediante la fórmula:

$$
(1-1 / 18) \times 100
$$

El rendimiento real (RR) se estimó mediante el seguimiento del desarrollo del cultivar Huinkul MAG en dos localidades, ambas ubicadas en el Partido de Balcarce. Una de ellas, denominada "INTA", corresponde al campo de la Estación Experimental Agropecuaria de Balcarce del Instituto Nacional de Tecnología Agropecuaria, y la otra, "San Agustín" ubicada en la chacra de la firma Nestlé S.A. en la localidad homónima.

En INTA se plantó el 9/10 y el 10/11/86 con una densidad de 67000 pedazos de tubérculos-semillas de papa ha ${ }^{-1}$, mientras que en San Agustín se plantó el 29/09 y el 19/10/86 con una densidad de 71000 pedazos ha $^{-1}$.

Las labores culturales pre y post-plantación se hicieron según la modalidad de la zona. A la plantación se fertilizó con 80 y 160 kg.ha- ${ }^{-1}$ 
de fosfato diamónico (18-46-0) en INTA y San Agustín, respectivamente; y se pulverizó contra gusanos del suelo con Heptacloro en la dosis recomendada. Durante el ciclo del cultivo, se pulverizó contra enfermedades fungosas a razón de 3 a 4 veces por mes; en ambos lugares se practicó riego suplementario por aspersión para mantener el nivel de humedad del suelo por encima del $50 \%$ de agua útil. En las Figuras 2 y 3 se presentan para el ciclo 1986/87, la evolución de la evapotranspiración potencial del cultivo (ETC) (7), las precipitaciones y los riegos efectuados.

Cada 15 días y para todas las situaciones mencionadas se cosecharon al azar 4 muestras de $2.25 \mathrm{~m}^{2}$ (2 surcos de $1.5 \mathrm{~m}$ de longitud). En cada cosecha se separó el follaje, tallos aéreos y tubérculos, registrándose peso fresco, sin incluir raíces. El peso seco de la parte aérea se obtuvo secando las muestras a $60^{\circ} \mathrm{C}$ en estufa con circulación de aire forzado hasta peso constante. Para estimar materia seca (M. S.) de tubérculos, se secaron a $80^{\circ} \mathrm{C}$ hasta peso constante alícuotas de 25-35 g (2 repeticiones por muestra) previamente trituradas y homogenizadas. Se registró también peso seco del material senescente.

En cada muestreo se realizaron mediciones de área foliar con un medidor AAC-400 HAYASHI DENKOH.

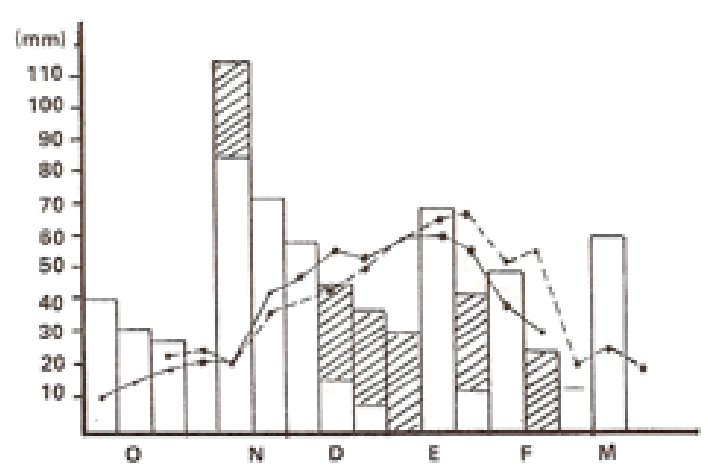

FIGURA 2. Valores decádicos de precipitación $\square$; riegos WIZA y evapotranspiración del cultivo (ETC) 


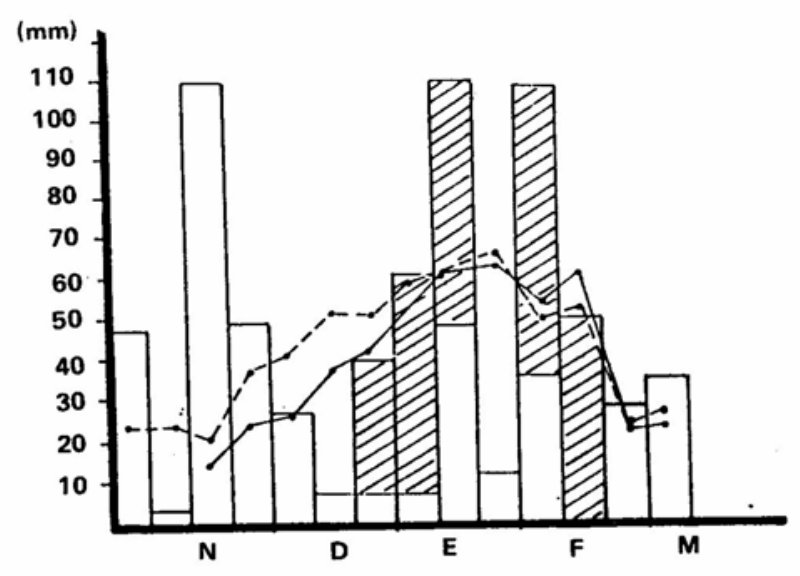

FIGURA 3. Valores decádicos de precipitación $\square$; riegos שa evapotranspiración del cultivo (ETC) para las dos fechas de plantación en INTA.

\section{RESULTADOS Y DISCUSIÓN}

En la Figura 4 se presentan las curvas de crecimiento del cultivo para la segunda fecha de plantación (19/10/86) que tuvo los mayores rendimientos reales, tanto en M. S. total como M. S. de tubérculos.

El RRP ${ }^{*}$ calculado según el modelo de Van der Zaag fue de $21.9 \mathrm{t}$ de M. S. (aproximadamente $88 \mathrm{t}$ en peso de tubérculo fresco). Este valor es el $12 \%$ menor al cálculo para los Países Bajos (20). La diferencia radica principalmente en los menores valores que se tomaron en la curva de intercepción ideal para Balcarce, y en las pérdidas respiratorias por temperaturas más altas durante el período del cultivo. El valor del Potencial de Balcarce es levemente menor a los estimados para Norteamérica y Europa Occidental (90-100 t de tubérculo en peso fresco) y superior a los estimados para las regiones tropicales y subtropicales $(40-60 \mathrm{t})(20,21)$.

\footnotetext{
*El detalle de los cálculos de los rendimientos potenciales y esperados se presentan en Cantos de Ruiz (5).
} 

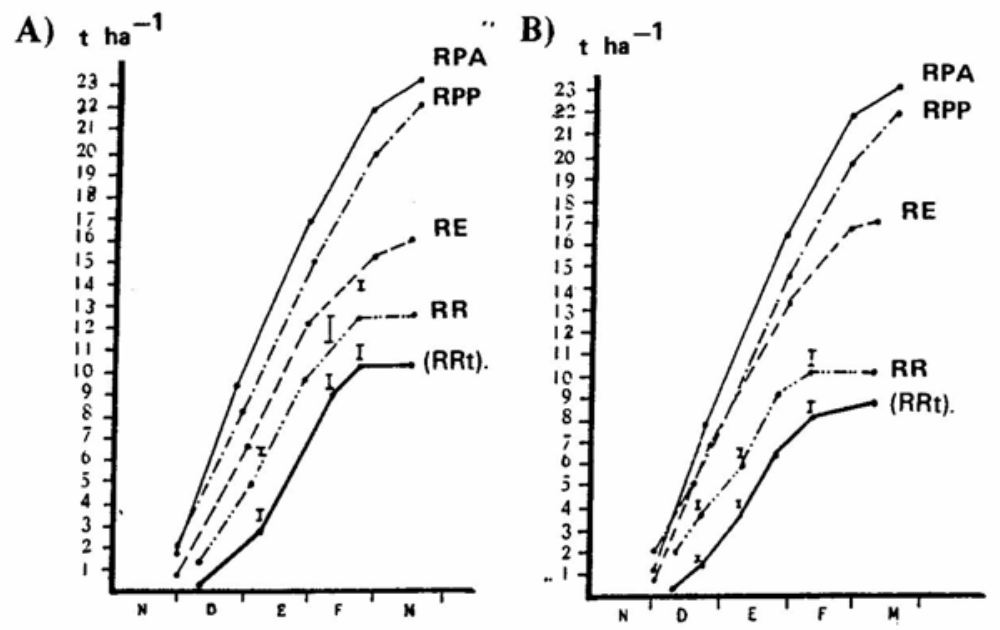

FIGURA 4. Producción de materia seca para papa cv. Huinkul MAG plantada en Balcarce. Rendimiento potencial promedio total (RPP); rendimiento potencial anual total (RPA); rendimiento esperado total (RE); rendimiento real total y rendimiento real de tubérculos (RRt).
A) Plantación del 19/10/86 en San Agustín.
B) Plantación del 19/10/86 en INTA.

Las barras corresponden al desvío estándar de las medias 
El RPA* para 1986/87 fue mayor que el promedio histórico debido principalmente a los altos valores de Heliofanía relativa de diciembre y enero y a las temperaturas más frescas de diciembre en el ciclo mencionado.

El RE* para esta fecha fue de $16.4 \mathrm{t}$ de materia seca total por hectárea (promedio de ambas localidades).

En el campo, el $\mathrm{RR}^{* *}$ promedio en esta fecha fue aproximadamente de 11.3 t de M.S. total.ha ${ }^{-1}$ que representa el $48 \%$ del RPA (Tabla 1). Este porcentaje se encuentra muy cercano a los obtenidos en los Países Bajos y al del Estado de Washington (USA) cuyas relaciones RR/RP son del 40\% (20). Esta relación da idea del elevado nivel técnico que tienen algunos productores de este cultivo. Los valores alcanzados en el experimento serían los esperados en Balcarce para una buena condición de manejo del cultivo.

Para dilucidar los factores responsables de la diferencia entre el RR y el RPA, en las Tablas 1 y 2 se presentan algunas relaciones entre los distintos tipos de rendimiento. Del cociente RER/RPA surge el grado de responsabilidad que tuvo la cobertura real en la disminución del rendimiento. Por otro lado, la capacidad productiva del follaje expresa como RR/RE, pues en ambos casos la curva de intercepción de RFA es la misma. El IC real relacionado con el valor tomado para papa (0.8) por Zaag y otros autores, refleja las características que tuvo la partición de asimilados a tubérculos en el cultivo real. Corrigiendo sucesivamente al RPA por estos factores, se obtiene la diferencia entre el máximo rendimiento alcanzable y lo que produjo el cultivo.

En San Agustín, para la fecha media, el factor que más incidió en la disminución del rendimiento fue cobertura (33\%) (Tabla 2). Hubo un desarrollo inicial muy lento del área foliar que produjo una diferencia importante entre las curvas de intercepción real e ideal (Figura 5a). Posiblemente las limitaciones fueron temperaturas bajas, pues el cultivo creció en una parte baja del campo. Varios autores indican que las bajas temperaturas resultan limitantes para el desarrollo foliar inicial $(2,17)$. Esta situación se observa en la diferencia entre las tasas de crecimiento esperada y potencial en noviembre y principios de diciembre (Figura 6a). Por otro lado, el riego suministrado no alcanzó a cubrir la gran demanda evaporativa de diciembre y enero, lo cual motivó un déficit hídrico que limitó el desarrollo foliar $(3,14)$ y redujo la productividad del follaje $(4,8,12,13)$.

\footnotetext{
* El detalle de los cálculos de los rendimientos potenciales y esperados se presentan en Cantos de Ruiz (5).

** El RR no incluye raíces.
} 
TABLA 1 .Rendimientos potenciales esperados y reales y sus relaciones. 1986

\begin{tabular}{|c|c|c|c|c|c|c|c|c|c|c|}
\hline \multirow[b]{2}{*}{$\begin{array}{l}\text { Lugar y } \\
\text { Fecha }\end{array}$} & \multirow{2}{*}{$\begin{array}{c}\text { Rend. } \\
\text { Potencial } \\
\text { Promedio } \\
\text { (RPP) }\end{array}$} & \multirow{2}{*}{$\begin{array}{c}\text { Rend. } \\
\text { Potencial } \\
\text { Anual } \\
\text { (RPA) }\end{array}$} & \multirow{2}{*}{$\begin{array}{l}\text { Rend. } \\
\text { Esperado } \\
\text { (RE) }\end{array}$} & \multirow{2}{*}{$\begin{array}{l}\text { Rend. } \\
\text { Real } \\
\text { (RR) }\end{array}$} & \multirow{2}{*}{$\begin{array}{l}\text { Rend. Real } \\
\text { de } \\
\text { Tubérculo. } \\
\text { (RRT) }\end{array}$} & \multirow{2}{*}{$\begin{array}{l}\text { Índice } \\
\text { Cosecha } \\
\text { (\%) } \\
\text { (IC) }\end{array}$} & \multicolumn{4}{|c|}{$\%$} \\
\hline & & & & & & & RE/RPA & RR/RE & RR/RPA & RRT/RPAt* \\
\hline $\begin{array}{c}\text { 19/10 } \\
\text { S. Agustín }\end{array}$ & & & 15.9 & 12.4 & 10.2 & 82.4 & 67.4 & 78.0 & 52.4 & 54.0 \\
\hline INTA & & & 17.0 & 10.2 & 8.7 & 85.0 & 72.0 & 60.0 & 43.2 & 46.1 \\
\hline PROMEDIO & 21.9 & 23.6 & 16.4 & 11.3 & 9.5 & 83.7 & 69.7 & 69.0 & 47.9 & 50.1 \\
\hline $29 / 09$ & & & & & & & & & & \\
\hline S. Agustín & & & 17.0 & 10.9 & 7.1 & 64.9 & 72.0 & 64.1 & 46.2 & 37.6 \\
\hline 10/11 INTA & & & 14.8 & 9.5 & 6.9 & 72.9 & 62.7 & 64.2 & 40.3 & 36.5 \\
\hline
\end{tabular}

* $\quad$ RPAt = RPA en M.S. de tubérculos (RPA x 0.8) 
TABLA 2. Contribución de intercepción de radiación-eficiencia de uso de la radiación e Índice de cosecha—a la diferencia entre los rendimientos reales y el potencial. Estos factores no son aditivos.

\section{Factores Responsables}

\section{Fecha de plantación/lugar}

$$
\begin{aligned}
& \text { Intercep. de RFA } \\
& \text { (RE/RPA-1)×100 }
\end{aligned}
$$
Producción de follaje (RR/RE - 1)×100

Indice de cosecha $(\mathrm{IC} / 0.8-1) \times 100$

$19 / 10 / 86$

$\begin{array}{lccc}\text { San Agustín } & -32.6 & -22.0 & +3.0 \\ \text { INTA } & -28.0 & -40.0 & -31.0 \\ \text { PROMEDIO } & -30.0 & & +6.3 \\ \text { 29/09/86 } & & -35.9 & -18.9 \\ \text { San Agustín } & -28.0 & -35.8 & -8.9 \\ \text { 10/11/86 } & & & \end{array}$

\section{*RFA= Radiación fotosintética activa}


En consecuencia, las tasas reales fueron menores a las esperadas para la cobertura existente y éstas, menores a las potenciales (Figura 6a). A partir de enero se superó el déficit, como lo indica la relación entre las tasas reales y esperadas; pero la intercepción disminuyó con respecto a la ideal, debido entre otros factores al escaso desarrollo foliar (Figura 5a).
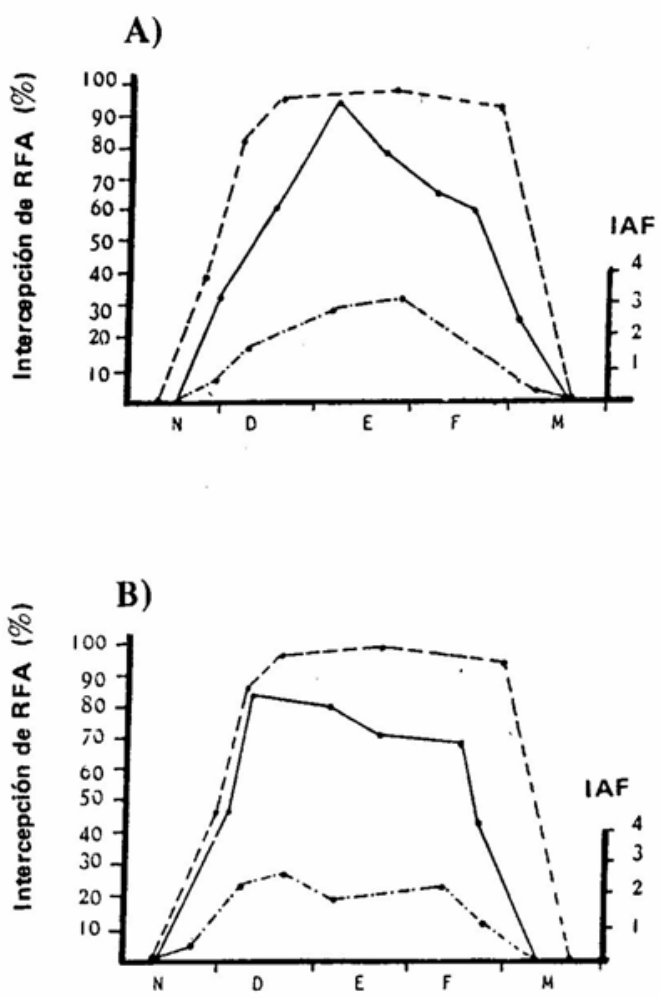

FIGURA 5. Radiación fotosintéticamente activa (RFA) interceptada por el cultivo de la papa, en porcentajes (Ideal estimada --_-- y Real media-) y evolución del índice de área foliar (IAF) (.........).
A) Plantación del 19/10/86 en San Agustín.
B) Plantación del 19/10/86 en INTA.

El desvío estándar de las medias osciló entre 0.1 y 0.7 para IAF y entre 1.3 y 7.7\% para intercepción de RFA. 
A)

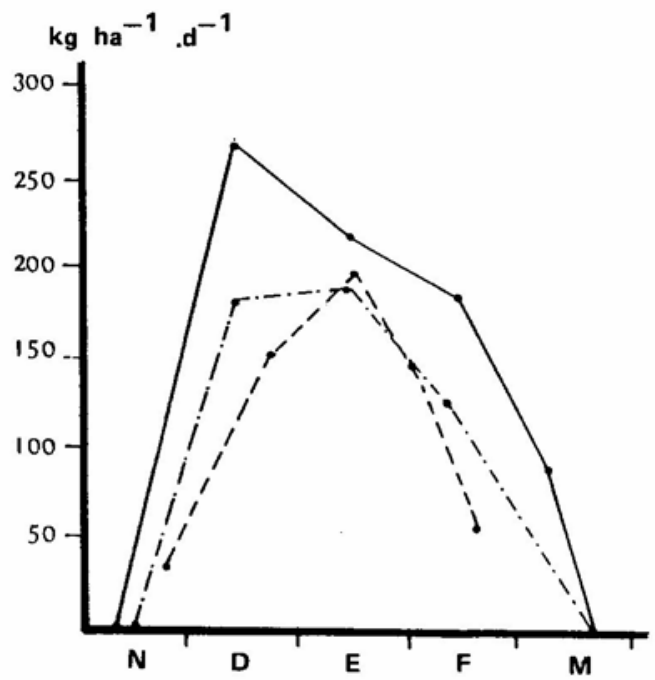

B)

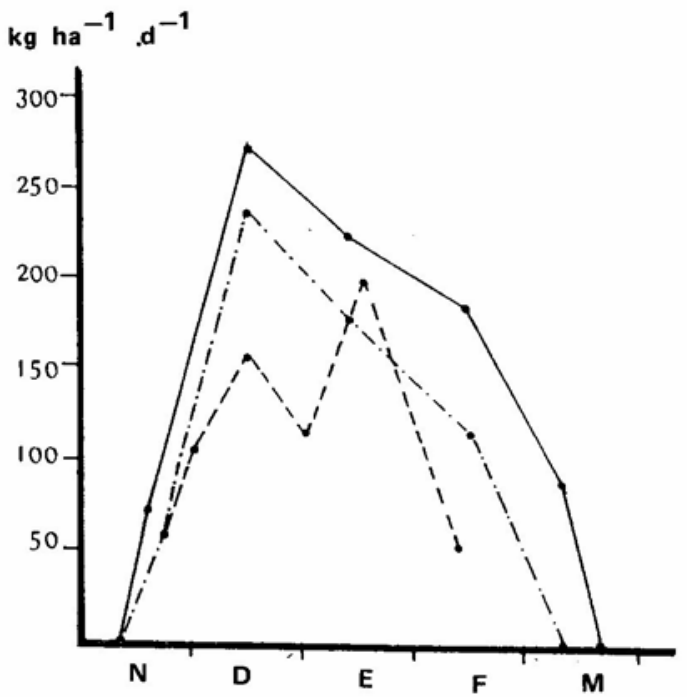

FIGURA 6. Evolución de las tasas de crecimiento potenciales esperadas - - - - - . - - . y reales $-\ldots-\ldots$ para la plantación del 19/10/86.
A) San Agustín.
B) INTA 
Para la misma fecha de plantación en INTA, el cultivo tuvo inicialmente un desarrollo similar al esperado (Figuras $4 b$ y $5 b$ ), pero la falta de agua de diciembre frenó la tendencia creciente del follaje y la tasa de crecimiento (Figura 6b). Los riegos no fueron tan regulares como en San Agustín y el aporte de lluvias en diciembre y enero también fue menor, por lo tanto, este cultivo sufrió en mayor medida la limitación de agua. Lo anterior se manifiesta en la diferencia entre las tasas de crecimiento reales y esperadas (Figura 6b). El déficit hídrico afectó principalmente la productividad del follaje sin afectar la cobertura inicial, pues en ese momento el cultivo tenía más de $80 \%$ de cobertura. Como resultado, la producción tuvo pérdidas del 40\% debido a ineficiencias en el uso de la radiación interceptada (Tabla 2). Las diferencias de fin de ciclo pueden deberse a intercepción de radiación por partes no activas y a pérdidas de materia seca.

Aunque la cobertura fue inicialmente mayor que en San Agustín, el escaso desarrollo foliar (IAF = 3) junto a enfermedades de fin de ciclo provocaron caídas en cobertura temprana. Debido al factor cobertura, el rendimiento esperado disminuyó el $28 \%$ con respecto al potencial.

EI IC en ambas localidades fue superior a 0.8 (Tabla 1) debido a que la fecha óptima no presentó problemas en la participación de asimilados.

En promedio, esta fecha intermedia tuvo pérdidas de rendimiento debido a fallas en la intercepción (30\%) y a menor eficiencia de uso de la radiación interceptada (31\%).

El plantar fuera de la época óptima de plantación trajo como consecuencia una disminución de los RR (Tabla 1).

En San Agustín para la fecha temprana se observaron efectos de bajas temperaturas y déficits hídricos en forma similar a lo acontecido para la fecha óptima en la misma localidad. Se interpreta que la menor productividad del follaje y el menor índice de cosecha obtenidos (Tabla 2) son producto de un retrocontrol sobre la fuente fotosintética por parte de los pocos destinos (tubérculos) formados como consecuencia de una tuberización temprana en siembras tempranas $(10,12)$.

Para la fecha tardía en INTA, el déficit hídrico produjo bajos valores de productividad del follaje, al igual que en la fecha óptima para la misma localidad. Debido a que el déficit hídrico tomó al cultivo en etapas ontogénicas más tempranas, y a que las condiciones de fin de ciclo provocaron una abrupta senescencia en esta fecha 
tardía, la intercepción de radiación fue la más pobre de todas las situaciones estudiadas (Tabla 2). Las condiciones de fin de ciclo impidieron al cultivo llenar los destinos predeterminados obteniendo un bajo índice de cosecha (Tablas 1 y 2) y escaso porcentaje de materia seca en tubérculos (5).

Los resultados obtenidos permiten identificar algunos factores cuyo manejo podría mejorar el cultivo de papa semitardía en Balcarce.

Las fechas de plantación intermedias no presentan problemas de partición de materia seca, pues los IC obtenidos son superiores a 0.8.

El cultivo es sumamente sensible a déficits hídricos, lo cual se puede producir a pesar de contar con riegos periódicos. El déficit hídrico ocasiona bajo desarrollo del IAF y poca cobertura, baja productividad del follaje y escasa duración del área foliar. Con riegos más frecuentes se obtendría más follaje y mayor duración del área foliar y se solucionaría el problema de ineficiencia de uso de la radiación interceptada (prueba de ésto es que las tasas reales igualen a las esperadas cuando los riegos o precipitaciones son suficientes). Con resultados de ensayos de riego llevados a cabo en INTA, Balcarce, durante 3 años (Suero y Travasso, datos no publicados) se estimó intercepción de radiación a partir de valores de IAF. Para ello se estableció, con nuestros datos, una relación cuadrática entre la evolución del IAF y el porcentaje de radiación que interceptó el cultivo. La ecuación tuvo muy buen ajuste $\left(R^{2}=0.94\right)$ y la curva coincidió con la obtenida en papa por otros autores (11). Luego, con los datos obtenidos de intercepción, se aplicó el modelo para cada año. El tratamiento con riego invariablemente alcanzó valores de eficiencia o productividad del follaje cercanos al 100\% y a una mayor duración del área foliar.

Para evitar las consecuencias de bajas temperaturas sobre el desarrollo foliar inicial, se debería poner especial cuidado en la selección de potreros ubicados en las partes altas, orientados preferencialmente hacia el Norte. Además, se presentan, como posibles soluciones para la senescencia temprana del follaje observada en el campo, el uso de papa-semilla joven, el aumento de suministro de nitrógeno, y el buen control de enfermedades y plagas durante y especialmente hacia el final del período de cultivo.

En resumen se pueden destacar las siguientes apreciaciones:

- El rendimiento potencial de la papa en Balcarce se estimó (según el modelo de Van der Zaag) en 88 t/ha ', de tubérculos, en peso fresco. 
- Los mejores rendimientos reales obtenidos alcanzaron el 53\% del valor potencial.

- En la fecha intermedia de plantación (19/10) se obtuvieron los mayores rendimientos. Los índices de cosecha fueron óptimos, y tanto la intercepción de radiación como la eficiencia de follaje fueron responsables de la diferencia entre el rendimiento real y el potencial.

- Adelantar o atrasar la fecha de plantación ocasionó mermas en la producción o biomasa a través principalmente de caídas en la productividad del follaje y en intercepción, respectivamente.

\section{REFERENCIAS BIBLIOGRÁFICAS}

1. Allen, E. J.; Scott, R. K. 1980. An analysis of growth of the potato crop. Jour. Agric. Sci., Camb. 94: 583-606.

2. Bodlaender, K. B. A. 1963. Influence of temperature, radiation and photoperiod on development and yield. p. 199-210. In: Ivins, J. D. and Milthorpe, F. L (eds.), The Growth of the Potato. London. Butterworths.

3. Boyer. J. S. 1970. Differing sensitivity of photosynthesis to low leaf water potentials in corn and soybean. Plant Physiology 46: 236-239.

4. Campbell, M. D.; Campbell, G. S ; Kunkel, K.; Papendick. R.J. 1976. A model describing soil-plant-water relations for potatoes. Amer. Potato J. 53: $431-444$.

5. Cantos de Ruiz, S. 1988. Rendimiento potencial del cultivo de papa en Balcarce. Causas que limitan la productividad real. Tesis Unidad Integrada Balcarce. Buenos Aires.

6. Della Vedova, O.; Mehrbald, M. H. 1984. Sistema de información para el plan nacional de abastecimiento de productos e insumos de origen agropecuario. PAPA: Estructura Regional y Destino de la Producción Nacional. Serie: Informes por Producto. No. 15. Sec. de Est. de Agric, y Gan. INTA.

7. Doorenbos, J.; Pruitt, W. O. 1977. Crop water requeriments. Irrigation and Drainage, paper No. 24, FAO. 144 p.

8. Epstein, E.; Grant, W. J. 1973. Water stress relations of the potato plant under field conditions. Agron. J. 66: 400-404.

9. Goundriaan, J.; Van Laar, H H. 1978. Calculations of daily total of the gross $\mathrm{CO}^{2}$ assimilation of leaf canopies. Neth. J. Agric. Sci. 26: 373-382.

10. Ivins, J. D.; Bremner, P. M. 1965. Outlook in Agriculture 4: 211-217. 
11. Khurana, S. C.; Mc Laren, J. S. 1982. The influence of leaf area, light interception and season on potato growth and yield. Potato Res. 25: 329342.

12. Milthorpe, F. F. ; Moorby, J. 1975. Potato. In: Evans, L. T. (ed.), CropPhysiology. Cambr. Univ. Press.

13. Moorby, J.; Munns, R.; Walcott, J. 1975. Effect of water déficit on photosyn-thesis and tuber metabolism in potatoes. Aus. J. Plant Phys. 2: 323-333.

14. Munns, R.; Pearson, C. J. 1974. Effects of water deficit on traslocation of carbohydrate in Solanum tuberosum. Aust. J. Plant Phys. 1: 529-537.

15. Sibma, L. 1969. Relation between total radiation and yield in the Netherlands. Neth. J. Agrie. Sci. 18: 125-131.

16. Sibma, L. 1977. Maximization of arable crop yields in the Netherland. Neth. J. Agrie. Sci. 25: 278-287.

17. Slater, J. W. 1968. The effect of night temperature on tuber initiation of the potato. Eur. Potalo J. 11: 14-22.

18. Wit, C. T. de. 1965. Photosynthesis of leaf canopies. Agric. Research Reports No. 663. Pudoc, Wageningen. 57 p.

19. Zaag, D. E. van der. 1984. Relability and significance of a simple method of estimating the potential yield of the potato crop. Potato Res. 27: 51 -73.

20. Zaag, D. E. van der. 1985. Some useful calculations regarding the potato crop. International Potato Course. I. A. C. Wageningen $43 \mathrm{p}$.

21. Zaag, D. E. van der; Burton, W. G. 1978 Potential yield of the potato crop and its limitations. 7lh. Triennial Conference E. A. P. R. Warsen, Poland. p. 7-22. 